\title{
Water-Protein Interactions: The Secret of Protein Dynamics
}

\author{
Silvia Martini, ${ }^{1,2}$ Claudia Bonechi, ${ }^{1,2}$ Alberto Foletti, ${ }^{3}$ and Claudio Rossi ${ }^{1,2}$ \\ ${ }^{1}$ Department of Biotechnology, Chemistry and Pharmacy, University of Siena, Via Aldo Moro 2, 53100 Siena, Italy \\ ${ }^{2}$ Centre for Colloid and Surface Science (CSGI), University of Florence, Via della Lastruccia 3, 50019 Sesto Fiorentino, Italy \\ ${ }^{3}$ Laboratory of Applied Mathematics and Physics, Department of Innovative Technologies-DTI, \\ University of Applied Sciences of Southern Switzerland (SUPSI), Manno, CH 6928, Switzerland \\ Correspondence should be addressed to Claudio Rossi; claudio.rossi@unisi.it
}

Received 31 January 2013; Accepted 15 April 2013

Academic Editors: J. Lipfert and G. Tresset

Copyright (c) 2013 Silvia Martini et al. This is an open access article distributed under the Creative Commons Attribution License, which permits unrestricted use, distribution, and reproduction in any medium, provided the original work is properly cited.

\begin{abstract}
Water-protein interactions help to maintain flexible conformation conditions which are required for multifunctional protein recognition processes. The intimate relationship between the protein surface and hydration water can be analyzed by studying experimental water properties measured in protein systems in solution. In particular, proteins in solution modify the structure and the dynamics of the bulk water at the solute-solvent interface. The ordering effects of proteins on hydration water are extended for several angstroms. In this paper we propose a method for analyzing the dynamical properties of the water molecules present in the hydration shells of proteins. The approach is based on the analysis of the effects of protein-solvent interactions on water protons NMR relaxation parameters. NMR relaxation parameters, especially the nonselective $\left(R_{1}^{\mathrm{NS}}\right)$ and selective $\left(R_{1}^{\mathrm{SE}}\right)$ spin-lattice relaxation rates of water protons, are useful for investigating the solvent dynamics at the macromolecule-solvent interfaces as well as the perturbation effects caused by the water-macromolecule interactions on the solvent dynamical properties. In this paper we demonstrate that Nuclear Magnetic Resonance Spectroscopy can be used to determine the dynamical contributions of proteins to the water molecules belonging to their hydration shells.
\end{abstract}

\section{Introduction}

Water-protein interactions play an important role in driving the protein organization at the water interface [1-4]. Waterprotein interactions help to maintain flexible conformation conditions which are required for multifunctional protein recognition processes. The intimate relationship between the protein surface and hydration water can be analyzed by studying experimental water properties measured in protein systems in solution. In particular, proteins in solution modify the structure and the dynamics of the bulk water at the solute-solvent interface. The ordering effects of proteins are extended for several angstroms. This process results in a protein hydration shell in which water molecules have restricted dynamics with respect to the bulk water. The extent of interaction can be monitored studying the solvent parameters mostly affected by the presence of a large, slowly reorienting biomacromolecule [5-12]. NMR relaxation parameters, especially the nonselective $\left(R_{1}^{\mathrm{NS}}\right)$ and selective $\left(R_{1}^{\mathrm{SE}}\right)$ spin-lattice relaxation rates of water protons, are useful for investigating the solvent dynamics at the macromolecule-solvent interfaces as well as the perturbation effects caused by the water-macromolecule interactions on the solvent dynamical properties [13-25]. In this paper we demonstrate that Nuclear Magnetic Resonance Spectroscopy can be used to determine the dynamical contribution of the biomacromolecules to the water molecules belonging to their hydration shells. In a globular protein solution, three different water environments are present, that is, the buried water molecules (which are integrant part of the protein structure and cannot be removed even during protein crystallization) [3, 4], the water hydration shell around the protein, and the bulk water. The present investigation analyzes the dynamical properties of the water molecules present in the hydration shell around a protein system. Water proton relaxation rates have been used to investigate different systems and phenomena, and theoretical interpretations of the experimental results have been proposed [26-30]. Both 


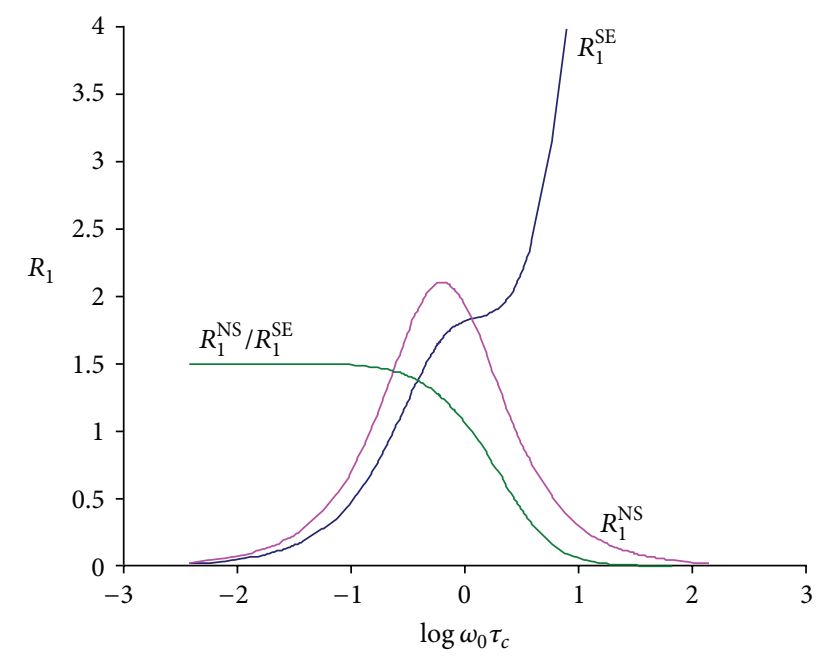

FIgURE 1: Dependence of selective and nonselective spin-lattice relaxation rates of the motion parameter $\omega_{0} \tau_{c}$.

the water proton spin-lattice relaxation rates $R_{1}^{\mathrm{NS}}$ and $R_{1}^{\mathrm{SE}}$ in solution are analyzed considering all possible sources of dipolar contributions arising from proton environments. From this analysis an equation for the calculation of ordering effect induced by the macromolecule on the hydration water was derived. In particular the average water rotational correlation time which characterizes water protons dynamics in the protein hydration shell was calculated. This information was then used for the calculation of the dimension of the long range ordering effect caused by the protein molecules on the hydration water.

\section{Theory}

Dipolar nonselective $R_{1}^{\mathrm{NS}}$ and selective $R_{1}^{\mathrm{SE}}$ spin-lattice relaxation rates have the following expressions [31-36]:

$$
\begin{gathered}
R_{1}^{\mathrm{NS}}=\sum \rho_{i j}+\sum \sigma_{i j}, \\
R_{1}^{\mathrm{SE}}=\sum \rho_{i j},
\end{gathered}
$$

where $\rho_{i j}$ is the direct self-relaxation rate and $\sigma_{i j}$ the "crossrelaxation" rates.

For any $i, j$ dipolar coupling $R_{1}^{\mathrm{NS}}$ and $R_{1}^{\mathrm{SE}}$ assume the explicit form:

$$
\begin{gathered}
R_{1}^{\mathrm{NS}}=\frac{3}{10} \frac{\gamma_{H}^{4} \hbar^{2}}{r_{i j}^{6}}\left[\frac{4 \tau_{c}}{1+4 \omega_{H}^{2} \tau_{c}^{2}}+\frac{\tau_{c}}{1+\omega_{H}^{2} \tau_{c}^{2}}\right], \\
R_{1}^{\mathrm{SE}}=\frac{1}{10} \frac{\gamma_{H}^{4} \hbar^{2}}{r_{i j}^{6}}\left[\frac{3 \tau_{c}}{1+\omega_{H}^{2} \tau_{c}^{2}}+\frac{6 \tau_{c}}{1+4 \omega_{H}^{2} \tau_{c}^{2}}+\tau_{c}\right],
\end{gathered}
$$

where $\hbar$ is the reduced Plank's constant, $\omega_{H}$ is the proton magnetogyric ratio and Larmor frequency, respectively, $r_{i j}$ is the internuclear distance, and $\tau_{c}$ is the effective correlation time which modulates the $i-j$ magnetic interaction.

The dependence of selective and nonselective spin-lattice relaxation rates of the motion parameter $\omega_{0} \tau_{c}$ is reported in Figure 1.

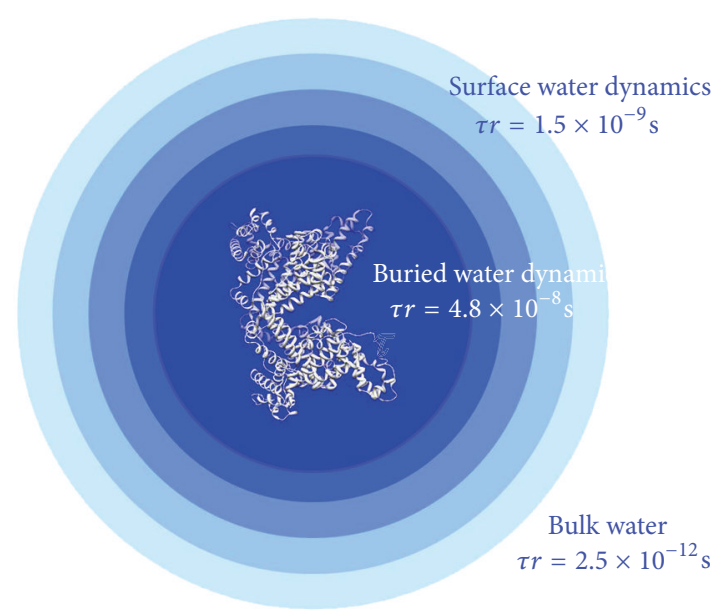

FIGURE 2: Effect of the ordering effect of proteins on water. Three water environments defined by their dynamical properties can be observed: bulk, surface, and buried water environments.

In pure water, the water nonselective $w R_{1}^{\mathrm{NS}}$ and selective $w R_{1}^{\mathrm{SE}}$ spin-lattice relaxation rates are

$$
\begin{aligned}
& w R_{1}^{\mathrm{NS}}=\sum \rho_{w w}+\sum \sigma_{w w}, \\
& w R_{1}^{\mathrm{SE}}=\sum \rho_{w w}+\sum \sigma_{w w},
\end{aligned}
$$

where $\rho_{w w}$ and $\sigma_{w w}$ are the water direct and cross-relaxation rate contributions which result from water proton-proton intra- and intermolecular interactions.

In pure water both $w R_{1}^{\mathrm{NS}}$ and $w R_{1}^{\mathrm{SE}}$ assume the same value as the cross-relaxation term $\sigma_{w w}$ affects the selective and nonselective measurements equally.

In binary system (water-protein and/or polymer) we assume the distribution of water molecules as schematically represented by the model showed in Figure 2. Water molecules can be classified into three different categories according to their dynamical properties: (i) bulk water with a typical reorientational correlation time of the order of picoseconds; (ii) water present at the macromolecular surface which exhibits a partially restricted reorientational motion; (iii) buried water molecules. The dynamical properties of the water molecules in these conditions can be well represented by a distribution of correlation time values. These molecules are in fast chemical exchange with the microenvironments present at the protein surface and with the bulk water molecules. These long lived water molecules show dynamics which are mostly determined by the slow reorientation motion of the macromolecule with $\tau_{c}$ values typically of the order of $10^{-8}$ seconds. These molecules exhibit slow chemical exchange rate in the NMR time scale with the waters present at the macromolecular surface. The contribution of these water molecules to the observed spin-lattice relaxation rates is negligible due to their very low molar fraction.

Relaxometric studies have been used to determine the number and the dominant reorientational correlation time which is involved in the relaxation of water molecules buried in the macromolecular structure [37]. Nevertheless 
relaxometric experiments cannot monitor the dominant fluctuations which are involved in the relaxation of the water molecules present at the macromolecular surface. In fact this environment is characterized by water molecules which exhibit a distribution of the $\tau_{c}$ values and display fast chemical exchange with other waters of the same environment or with the bulk molecules. These are in fact the appropriate conditions for applying the selective and nonselective water spin-lattice relaxation methodologies.

In water-protein binary systems, under fast chemical exchange conditions between the free (bulk) and bound water, the changes observed in water spin-lattice relaxation rates with respect to the bulk water reflect the presence of water molecules with restricted dynamical reorientation. In these conditions nonselective $\left(w R_{1}^{\mathrm{NS}}\right)$ and selective $\left(w R_{1}^{\mathrm{SE}}\right)$ water spin-lattice relaxation rates assume different values as a consequence of a negative protein-water cross-relaxation contribution to $w R_{1}^{\mathrm{NS}}$ and $w R_{1}^{\mathrm{SE}}$. They are defined as

$$
w R_{1 \exp }=\chi_{b} R_{1 b}+\chi_{f} R_{1 f}
$$

where $w R_{1 \exp }$ is the experimental relaxation rate of water in the presence of the protein, $R_{1 b}$ and $R_{1 f}$ are the water relaxation rates of the pure bound and free environments, and $\chi_{b}$ and $\chi_{f}$ are the molar fraction of water in bound and bulk conditions.

$\chi_{f}$ of the free water molar fraction is assumed to be $\chi_{f}=$ $1-\chi_{b} \cong 1$.

At the bound site, in the presence of $\mathrm{D}_{2} \mathrm{O}>95 \%$, the residual water protons show a relaxation which is mainly dominated by the dipolar interactions with the nonexchangeable protein protons. Water-water interactions (both interand intra-) have a sufficient low frequency to be neglected so that

$$
\begin{gathered}
w R_{1 \exp }^{\mathrm{NS}}=w R_{1}^{\mathrm{NS}}+\chi_{b}\left(\sum \rho_{w p}+\sum \sigma_{w p}\right), \\
w R_{1 \exp }^{\mathrm{SE}}=w R_{1}^{\mathrm{SE}}+\chi_{b}\left(\sum \rho_{w p}\right) .
\end{gathered}
$$

Then the protein contribution to the water relaxation rates, $\Delta R_{1}$, can be calculated as

$$
\begin{gathered}
\Delta R_{1}^{\mathrm{NS}}=w R_{1 \exp }^{\mathrm{NS}}-w R_{1}^{\mathrm{NS}}=\chi_{b}\left(\sum \rho_{w p}+\sum \sigma_{w p}\right)=\chi_{b} R_{1 b}^{\mathrm{NS}}, \\
\Delta R_{1}^{\mathrm{SE}}=w R_{1 \exp }^{\mathrm{SE}}-w R_{1}^{\mathrm{SE}}=\chi_{b}\left(\sum \rho_{w p}\right)=\chi_{b} R_{1 b}^{\mathrm{SE}},
\end{gathered}
$$

where $R_{1 b}^{\mathrm{NS}}$ and $R_{1 b}^{\mathrm{SE}}$ are the relaxation rates of the water molecules present in the bound conditions.

Considering the dependence of the $R_{1}^{\mathrm{NS}} / R_{1}^{\mathrm{SE}}$ ratio on $\tau_{c}$ (see (2)), $\Delta R_{1}^{\mathrm{NS}} / \Delta R_{1}^{\mathrm{SE}}$ ratio allows the calculation of the $\tau_{c}$ value resulting from the average contribution of the distribution of motions that characterizes the water dynamics at the macromolecular surface. Equation (2) holds their own validity when a single correlation time value is replaced by a distribution function which considers all different fast exchanging microenvironments.
In fact

$$
\begin{aligned}
\frac{\Delta R_{1}^{\mathrm{NS}}}{\Delta R_{1}^{\mathrm{SE}}} & =\frac{\chi_{b} R_{1 b}^{\mathrm{NS}}}{\chi_{b} R_{1 b}^{\mathrm{SE}}}=\frac{R_{1 b}^{\mathrm{NS}}}{R_{1 b}^{\mathrm{SE}}} \\
& =\frac{12 \tau_{c 1} /\left(1+4 \omega_{H}^{2} \tau_{c 1}^{2}\right)+3 \tau_{c 1} /\left(1+\omega_{H}^{2} \tau_{c 1}^{2}\right)}{6 \tau_{c 1} /\left(1+4 \omega_{H}^{2} \tau_{c 1}^{2}\right)+3 \tau_{c 1} /\left(1+\omega_{H}^{2} \tau_{c 1}^{2}\right)+\tau_{c 1}}
\end{aligned}
$$

where $\tau_{c 1}$ represents a distribution function which considers all individual dynamics which modulate the relaxation. The calculated $\tau_{c}$ value may be not directly related to a physical meaning as the presence at the macromolecular surface of a specific dynamics defined by this value is not demonstrated. Nevertheless this experimentally determined parameter represents the average value which affects the dipolar waterprotein interactions at the macromolecular surface. This parameter assumes a value which has to be in between the protein $\tau_{c}$ reorientational motion $\left(\sim 10^{-8} \mathrm{~s}\right)$ and the solvent free tumbling reorientation $\left(\sim 10^{-12} \mathrm{~s}\right)$.

\section{Materials and Methods}

${ }^{1} \mathrm{H}-\mathrm{NMR}$ spectra were obtained on a Bruker AMX 400 spectrometer operating at $400 \mathrm{MHz}$. Spin-lattice relaxation rates were measured using the $\left(180^{\circ}-\tau-90^{\circ}-t\right)_{n}$ sequence. The $\tau$ values used for the selective and nonselective experiments were $0.01,0.02,0.04,0.06,0.08,0.1,0.2,0.4,0.8,1,1.5,2$, $3,4,5,7$, and 10 seconds. The $180^{\circ}$ selective inversion of the proton spin population was obtained with a selective perturbation pulse, generated by the decoupler channel. The selective spin-lattice relaxation rates were calculated using the initial slope approximation and subsequent three-parameter exponential regression analysis of the longitudinal recovery curves. The maximum experimental error in the relaxation rate measurements was $5 \%$.

Human albumin (molecular weight 66200 Dalton) was purchased from Sigma Chemical Co. All the solutions were obtained using $\mathrm{D}_{2} \mathrm{O}$ with a minimum content of deuterium of $99.9 \%$.

\section{Results and Discussion}

The theory presented in the previous section is supported by the experimental results obtained on human albumin system.

Water selective and nonselective spin-lattice relaxation rates as a function of protein concentrations are reported in Table 1 .

The proteins contribution to the water selective $\Delta R_{1}^{\mathrm{SE}}$ and nonselective $\Delta R_{1}^{\mathrm{NS}}$ relaxation for human albumin systems is shown in Figure 3. In this figure the fitting of the experimental results is also shown. As required by the theory, the calculated straight lines pass through the origin in the system under study. As shown in Figure 3, water selective spin-lattice relaxation rates assume a larger value with respect to the water nonselective spin-lattice relaxation rates, whose results are affected by the negative protein-water cross-relaxation contributions.

The ratio calculated from the proteins contribution to the water nonselective and selective relaxation rates, $\Delta R_{1}^{\mathrm{NS}} / \Delta R_{1}^{\mathrm{SE}}$, 
TABLE 1: Water non-selective and selective proton spin-lattice relaxation times as a function of the human albumin content at $298 \mathrm{~K}$. In the same table the protein contribution to the selective and non-selective proton spin-lattice relaxation rates $\Delta R_{1}^{\mathrm{SE}}$ and $\Delta R_{1}^{\mathrm{NS}}$ is also reported.

\begin{tabular}{|c|c|c|c|c|c|c|c|}
\hline $\begin{array}{l}\text { Albumin concentration } \\
\mathrm{mol} / \mathrm{L}\end{array}$ & $\begin{array}{l}\text { Albumin concentration } \\
\mathrm{mg} / \mathrm{mL}\end{array}$ & $\begin{array}{c}T_{1}^{\mathrm{NS}} \\
\mathrm{s} \\
\end{array}$ & $\begin{array}{c}T_{1}^{\mathrm{SE}} \\
\mathrm{S}\end{array}$ & $\begin{array}{l}R_{1}^{\mathrm{NS}} \\
\mathrm{s}^{-1}\end{array}$ & $\begin{array}{l}R_{1}^{\mathrm{SE}} \\
\mathrm{s}^{-1}\end{array}$ & $\begin{array}{c}\Delta R_{1}^{\mathrm{NS}} \\
\mathrm{s}^{-1}\end{array}$ & $\begin{array}{c}\Delta R_{1}^{\mathrm{SE}} \\
\mathrm{s}^{-1}\end{array}$ \\
\hline 0 & 0 & 10.10 & 10.30 & 0.099 & 0.097 & 0 & 0 \\
\hline $1.6 \times 10^{-5}$ & 1.0 & 8.30 & 6.45 & 0.120 & 0.155 & 0.021 & 0.058 \\
\hline $3.2 \times 10^{-5}$ & 2.0 & 7.10 & 4.55 & 0.141 & 0.220 & 0.042 & 0.123 \\
\hline $4.8 \times 10^{-5}$ & 3.0 & 6.15 & 3.60 & 0.163 & 0.278 & 0.064 & 0.181 \\
\hline $6.5 \times 10^{-5}$ & 4.0 & 5.50 & 2.90 & 0.182 & 0.345 & 0.083 & 0.248 \\
\hline $7.3 \times 10^{-5}$ & 4.5 & 5.10 & 2.70 & 0.196 & 0.370 & 0.097 & 0.273 \\
\hline $8.1 \times 10^{-5}$ & 5.0 & 4.70 & 2.45 & 0.213 & 0.408 & 0.114 & 0.311 \\
\hline $8.9 \times 10^{-5}$ & 5.5 & 4.45 & 2.30 & 0.225 & 0.435 & 0.126 & 0.338 \\
\hline $9.7 \times 10^{-5}$ & 6.0 & 4.15 & 2.10 & 0.241 & 0.476 & 0.142 & 0.379 \\
\hline $1.3 \times 10^{-4}$ & 8.0 & 3.55 & 1.66 & 0.282 & 0.602 & 0.183 & 0.505 \\
\hline $1.6 \times 10^{-4}$ & 10.0 & 3.10 & 1.40 & 0.323 & 0.714 & 0.202 & 0.559 \\
\hline
\end{tabular}

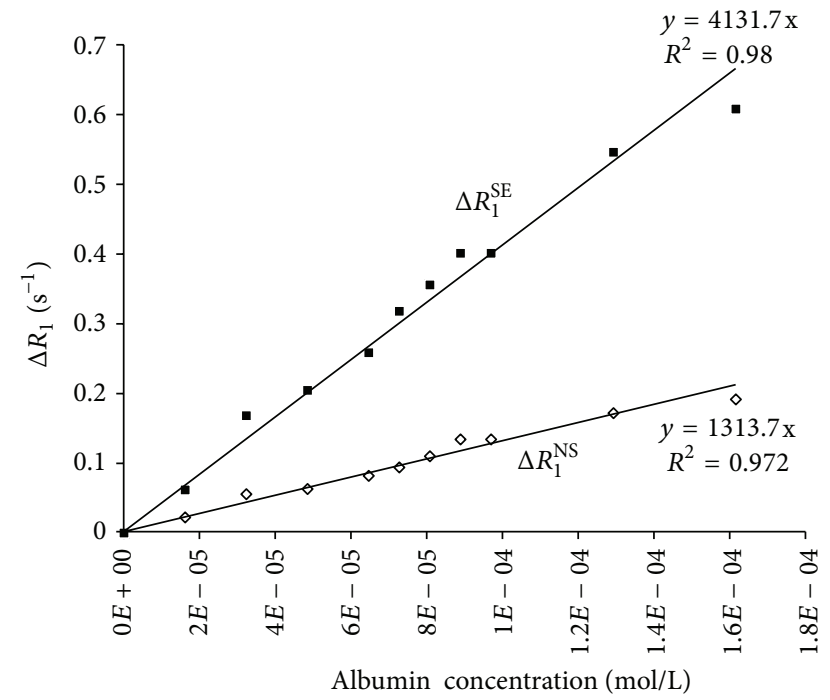

FIGURE 3: Nonselective and selective proton spin-lattice relaxation rates $\Delta R_{1}^{\mathrm{SE}}$ and $\Delta R_{1}^{\mathrm{NS}}$ as a function of the human albumin concentration.

assumes a value of 0.36 . The behavior of the $\Delta R_{1}^{\mathrm{NS}} / \Delta R_{1}^{\mathrm{SE}}$ ratio as a function of $\tau_{c}$ is reported in Figure 4 .

Using the previously computed $\Delta R_{1}^{\mathrm{NS}} / \Delta R_{1}^{\mathrm{SE}}$ ratio of 0.36 , an average reorientational correlation time of $1.5 \times 10^{-9} \mathrm{~s}$ was calculated for the water molecules in the protein hydration shell. In Figure 1 a summary of the water environment typical of protein systems in the case of human albumin is shown: bulk, buried, and hydration water. In the same figure the rotational correlation time values typical of each water environments are reported. The average water hydration correlation time previously computed was used to calculate the ordering effects of the protein on water molecules in the hydration shells at different distance from the protein surface. Assuming a spherical shape with a diameter of $70 \AA$, the volume of ten hydration spheres around human albumin was

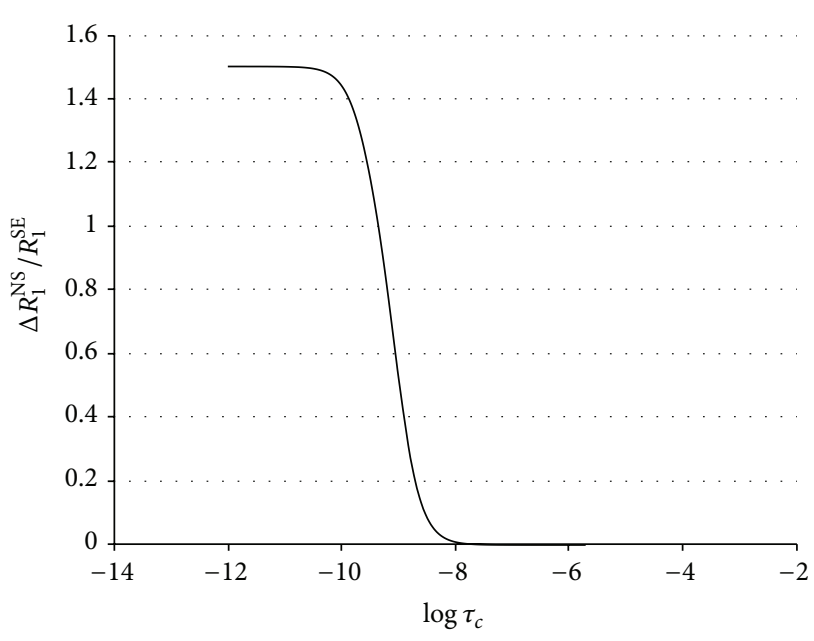

FIGURE 4: Computed values of $\Delta R_{1}^{\mathrm{NS}} / \Delta R_{1}^{\mathrm{SE}}$ ratio as a function of $\tau_{c}$ at a proton frequency of $400 \mathrm{MHz}$.

calculated. The number of water molecules in each hydration sphere was computed as well as the number of the total water molecules contained in the first ten hydration spheres. Assuming an exponential decay of the water correlation time from its value at the protein surface to the bulk conditions, the following equation was developed:

$$
\tau_{c(1,2, \ldots, 10)}=a+b e^{-k d}
$$

where $\tau_{c(1,2, \ldots, 10)}$ are the calculated correlation time values of the water molecules present in the first tenth hydration shell, $a=2.5 \times 10^{-12} \mathrm{~s}$ is the bulk water rotational $\tau_{\mathrm{c}}, b=4.8 \times$ $10^{-8} \mathrm{~s}$ is the buried water rotational $\tau_{c}, d$ is the hydration shell distance from the protein surface assumed here to range from 1 to $10 \AA, k$ is a constant which defines how strong the ordering effect of the protein on the water molecules. In Figure 4 the computed correlation times (calculated from equation (7)) of the water molecules in each of the first tenth hydration shells 


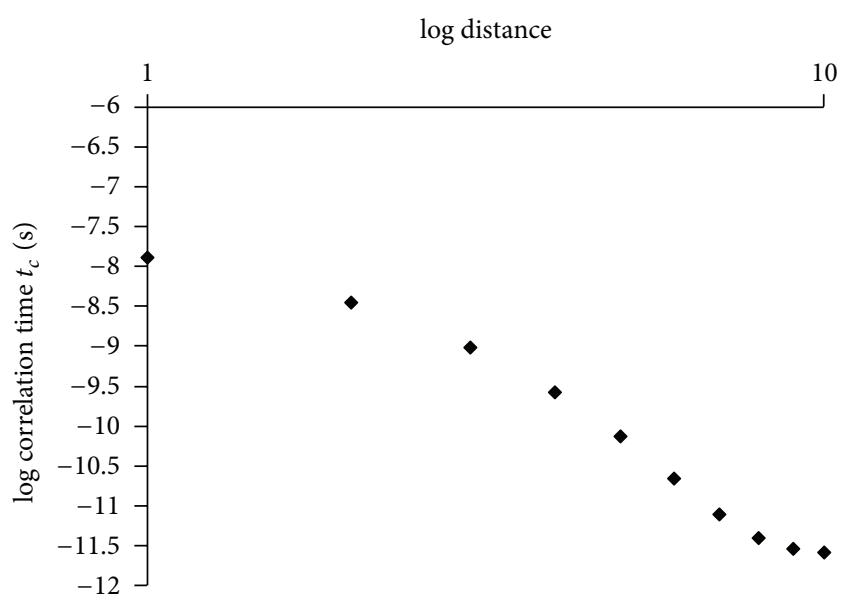

FIGURE 5: Computed values of the reorientational water correlation times typical of water molecules in the first tenth hydration shells around human albumin. Correlation time was calculated using equation (8) with $a=2.5 \times 10^{-12}, b=4.8 \times 10^{-8}$, and $k=1.3$. The average correlation time over the ten shells was calculated using the equation $\tau_{c \text { average }}=\sum_{i=1}^{10} \chi_{i} \tau_{c i}=1.5 \times 10^{-9} \mathrm{~s}$.

as a function of the distance $d$ are reported. The convergence between the experimental average reorientational correlation times of the water molecules in the protein hydration shells of $1.5 \times 10^{-9} \mathrm{~s}$ with the value computed on the basis of (8) was obtained for a $k$ equal to $1.3\left(\AA^{-1}\right)$. The long range ordering effect of the protein on the hydration water is extended at least to $8 \AA$ (Figure 5).

\section{Conclusions}

In diluted protein solutions, the bulk water proton relaxation shared the contributions from the water molecules in the protein hydration shell. These water molecules differ from the bulk water, mainly because of their correlation times, which is are short for bulk water and longer for the protein hydration waters. In slow motion conditions $\left(\omega_{0} \tau_{c} \gg 1\right.$, typical of the slow tumbling of protein molecules, these contributions are different: large and positive to $w R_{1}^{\mathrm{SE}}$ and negligible or absent to $w R_{1}^{\mathrm{NS}}$. This process makes $w R_{1}^{\mathrm{SE}}$ larger than $w R_{1}^{\mathrm{NS}}$ as showed in Figure 3. The analysis of both the selective and nonselective water spin-lattice relaxation rates allowed the calculation of the average effective correlation time for the water molecules at the water-protein interface. Moreover, using the assumption of an exponential decay of the rotational correlation time of the hydration water from its value at the protein surface to the bulk conditions, the long range ordering effect of the protein surface on the surrounded water molecules was calculated.

\section{Acknowledgment}

This work was partially supported by LIR srl.

\section{References}

[1] B. Halle, "Protein hydration dynamics in solution: a critical survey," Philosophical Transactions of the Royal Society B, vol. 359, pp. 1207-1224, 2004.

[2] S. Bone and R. Pethig, "Dielectric studies of protein hydration and hydration-induced flexibility," Journal of Molecular Biology, vol. 181, no. 2, pp. 323-326, 1985.

[3] K. Takano, Y. Yamagata, and K. Yutani, "Buried water molecules contribute to the conformational stability of a protein," Protein Engineering, vol. 16, no. 1, pp. 5-9, 2003.

[4] S. Fisher and C. S. Verma, "Binding of buried structural water increases the flexibility of proteins," Proceedings of the National Academy of Sciences of the United States of America, vol. 96, no. 17, p. $9613,1999$.

[5] V. P. Denisov and B. Halle, "Protein hydration dynamics in aqueous solution," Faraday Discussions, vol. 103, pp. 227-244, 1996.

[6] M. Vorob'ev, "Monitoring of water ordering in aqueous protein systems," Food Hydrocolloids, vol. 21, no. 2, pp. 309-312, 2007.

[7] N. Serge and S. N. Timasheff, "Protein-solvent preferential interactions, protein hydration, and the modulation of biochemical reactions by solvent components," Proceedings of the National Academy of Sciences of the United States of America, vol. 99, no. 15, pp. 9721-9726, 2002.

[8] G. Zaccai, "The effect of water on protein dynamics," Proceedings of the National Academy of Sciences of the United States of America, vol. 359, no. 1448, pp. 1269-1275, 2004.

[9] N. Bhattacharjee and P. Biswas, "Structure of hydration water in proteins: a comparison of molecular dynamics simulations and database analysis," Biophysical Chemistry, vol. 158, no. 1, pp. 73-80, 2011.

[10] M. Belotti, A. Martinelli, R. Gianferri, and E. Brosio, "A proton NMR relaxation study of water dynamics in bovine serum albumin nanoparticles," Physical Chemistry Chemical Physics, vol. 12, no. 2, pp. 516-522, 2010.

[11] M. Jasnin, A. Stadler, M. Tehei, and G. Zaccai, "Specific cellular water dynamics observed in vivo by neutron scattering and NMR," Physical Chemistry Chemical Physics, vol. 12, no. 35, pp. 10154-10160, 2010.

[12] D. I. Svergun, S. Richard, M. H. J. Koch, Z. Sayers, S. Kuprin, and G. Zaccai, "Protein hydration in solution: experimental observation by $\mathrm{x}$-ray and neutron scattering," Proceedings of the National Academy of Sciences of the United States of America, vol. 95, no. 5, pp. 2267-2272, 1998.

[13] R. G. Bryant, "The dynamics of water-protein interactions," Annual Review of Biophysics and Biomolecular Structure, vol. 25, pp. 29-53, 1996.

[14] N. Niccolai, L. Pogliani, and C. Rossi, "Water proton selective nuclear relaxation: a proof to investigate surface characteristics of biological materials," Chemical Physics Letters, vol. 110, no. 3, pp. 294-297, 1984.

[15] C. Rossi, A. Donati, and M. R. Sansoni, "Nuclear magnetic resonance as a tool for the identification of specific DNAligand interaction," Chemical Physics Letters, vol. 189, no. 3, pp. 278-280, 1992.

[16] C. Bonechi, A. Donati, M. P. Picchi, C. Rossi, and E. Tiezzi, "DNA-ligand interaction detected by proton selective and nonselective spin-lattice relaxation rate analysis," Colloids and Surfaces A, vol. 115, pp. 89-95, 1996.

[17] C. Rossi, S. Bastianoni, C. Bonechi, G. Corbini, P. Corti, and A. Donati, "Ligand-protein recognition studies as determined by 
nuclear relaxation analysis," Chemical Physics Letters, vol. 310, no. 5-6, pp. 495-500, 1999.

[18] N. Niccolai, C. Rossi, and E. Tiezzi, "Water proton spin-lattice relaxation behaviour in heterogeneous biological systems," Chemical Physics Letters, vol. 96, no. 2, pp. 154-156, 1983.

[19] G. Otting, E. Liepinsh, and K. Wuthrich, "Protein hydration in aqueous solution,” Science, vol. 254, no. 5034, pp. 974-980, 1991.

[20] C. Rossi, A. Donati, C. Bonechi et al., "Nuclear relaxation studies in ligand-macromolecule affinity index determinations," Chemical Physics Letters, vol. 264, no. 1-2, pp. 205-209, 1997.

[21] S. Martini, C. Bonechi, and C. Rossi, "Interaction of quercetin and Its conjugate quercetin 3-O- $\beta$-d-glucopyranoside with albumin as determined by NMR relaxation data," Journal of Natural Products, vol. 71, no. 2, pp. 175-178, 2008.

[22] S. Martini, C. Bonechi, M. Casolaro, G. Corbini, and C. Rossi, "Drug-protein recognition processes investigated by NMR relaxation data: a study on corticosteroid-albumin interactions," Biochemical Pharmacology, vol. 71, no. 6, pp. 858-864, 2006.

[23] S. Martini, C. Bonechi, and C. Rossi, "Interaction between vine pesticides and bovine serum albumin studied by nuclear spin relaxation data," Journal of Agricultural and Food Chemistry, vol. 58, no. 19, pp. 10705-10709, 2010.

[24] S. Martini, M. Consumi, C. Bonechi, C. Rossi, and A. Magnani, "Fibrinogen-catecholamine interaction as observed by NMR and fourier transform infrared spectroscopy," Biomacromolecules, vol. 8, no. 9, pp. 2689-2696, 2007.

[25] S. Martini, C. Bonechi, G. Corbini, and C. Rossi, "Determination of the modified "affinity index" of small ligands and macromolecular receptors from NMR spin-lattice relaxation data," Chemical Physics Letters, vol. 447, no. 1-3, pp. 147-153, 2007.

[26] R. Damadian, "Tumor detection by nuclear magnetic resonance," Science, vol. 171, no. 3976, pp. 1151-1153, 1971.

[27] V. R. Mathur-De, "The NMR studies of water in biological systems," Progress in Biophysics and Molecular Biology, vol. 35, pp. 103-134, 1980.

[28] D. E. Woessner, "Nuclear magnetic relaxation and structure in aqueous heterogenous systems," Molecular Physics, vol. 34, no. 4, pp. 899-920, 1977.

[29] S. H. Koenig, R. D. Brown III, and R. Ugolini, "A unified view of relaxation in protein solutions and tissue, including hydration and magnetization transfer," Magnetic Resonance in Medicine, vol. 29, no. 1, pp. 77-83, 1993.

[30] R. G. Bryant and W. M. Shirley, "Dynamical deductions from nuclear magnetic resonance relaxation measurements at the water-protein interface," Biophysical Journal, vol. 32, no. 1, pp. 3-16, 1980.

[31] F. Bloch, "Generalized theory of relaxation," Physical Review, vol. 105, no. 4, pp. 1206-1222, 1957.

[32] I. Solomon, "Relaxation processes in a system of two spins," Physical Review, vol. 99, no. 2, pp. 559-565, 1955.

[33] J. H. Noggle and R. E. Shirmer, The Nuclear Overhauser Effect, Academic, New York, NY, USA, 1971.

[34] C. Rossi, S. Bastianoni, C. Bonechi, G. Corbini, P. Corti, and A. Donati, "Ligand-protein recognition studies as determined by nuclear relaxation analysis," Chemical Physics Letters, vol. 310, no. 5-6, pp. 495-500, 1999.

[35] D. Neuhaus and M. Williamson, The Nuclear Overhauser Effect in Structural and Conformational Analysis, VCH Publisher, New York, NY, USA, 1989.
[36] R. Freeman, H. D. W. Hill, B. L. Tomlinson, and L. D. Hall, "Dipolar contribution to NMR spin-lattice relaxation of protons," Journal of Chemical Physics, vol. 61, no. 11, pp. 44664473, 1974.

[37] S. Kiihne and R. G. Bryant, "Protein-bound water molecule counting by resolution of (1)H spin-lattice relaxation mechanisms," Biophysical Journal, vol. 78, no. 4, pp. 2163-2169, 2000. 

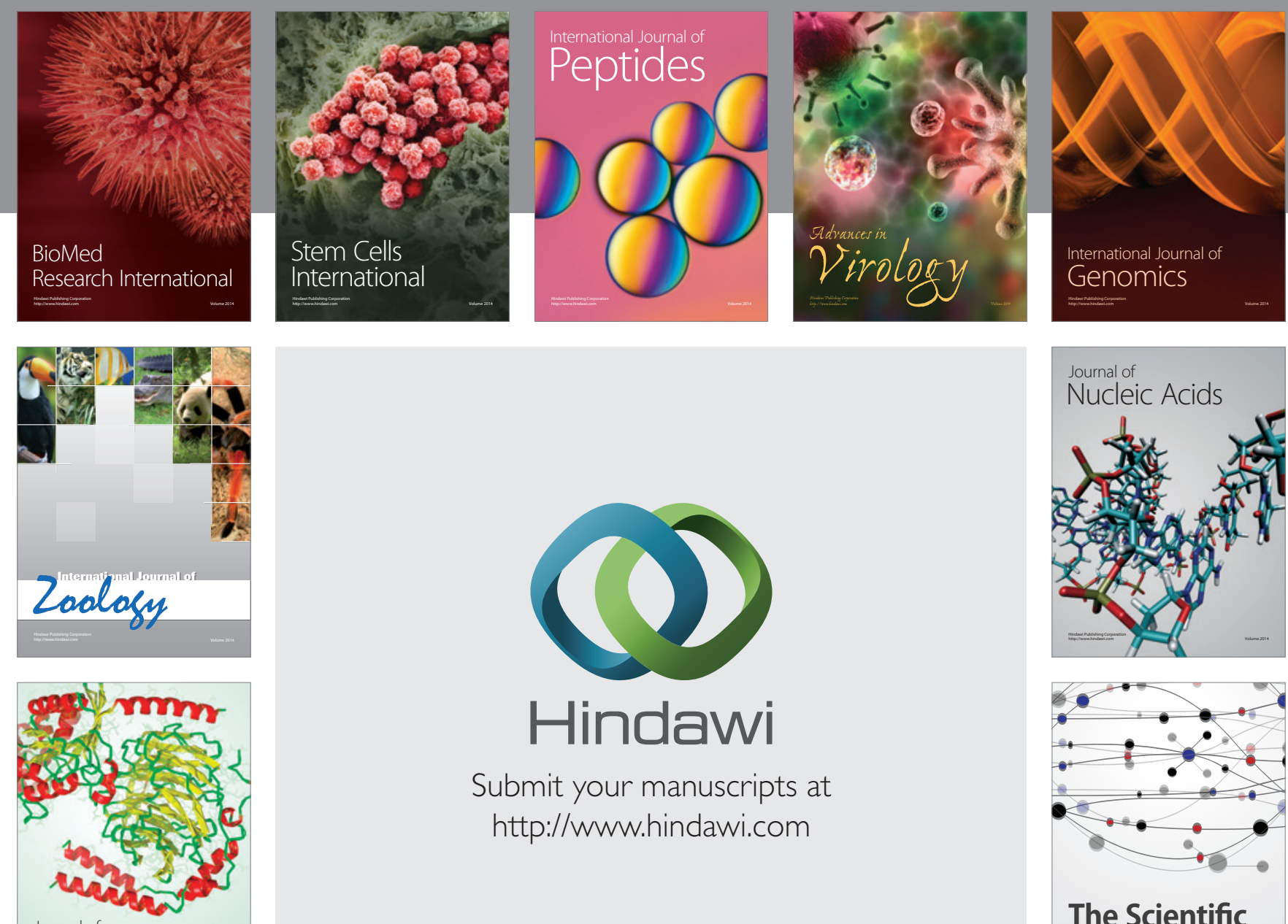

Submit your manuscripts at

http://www.hindawi.com

Journal of
Signal Transduction
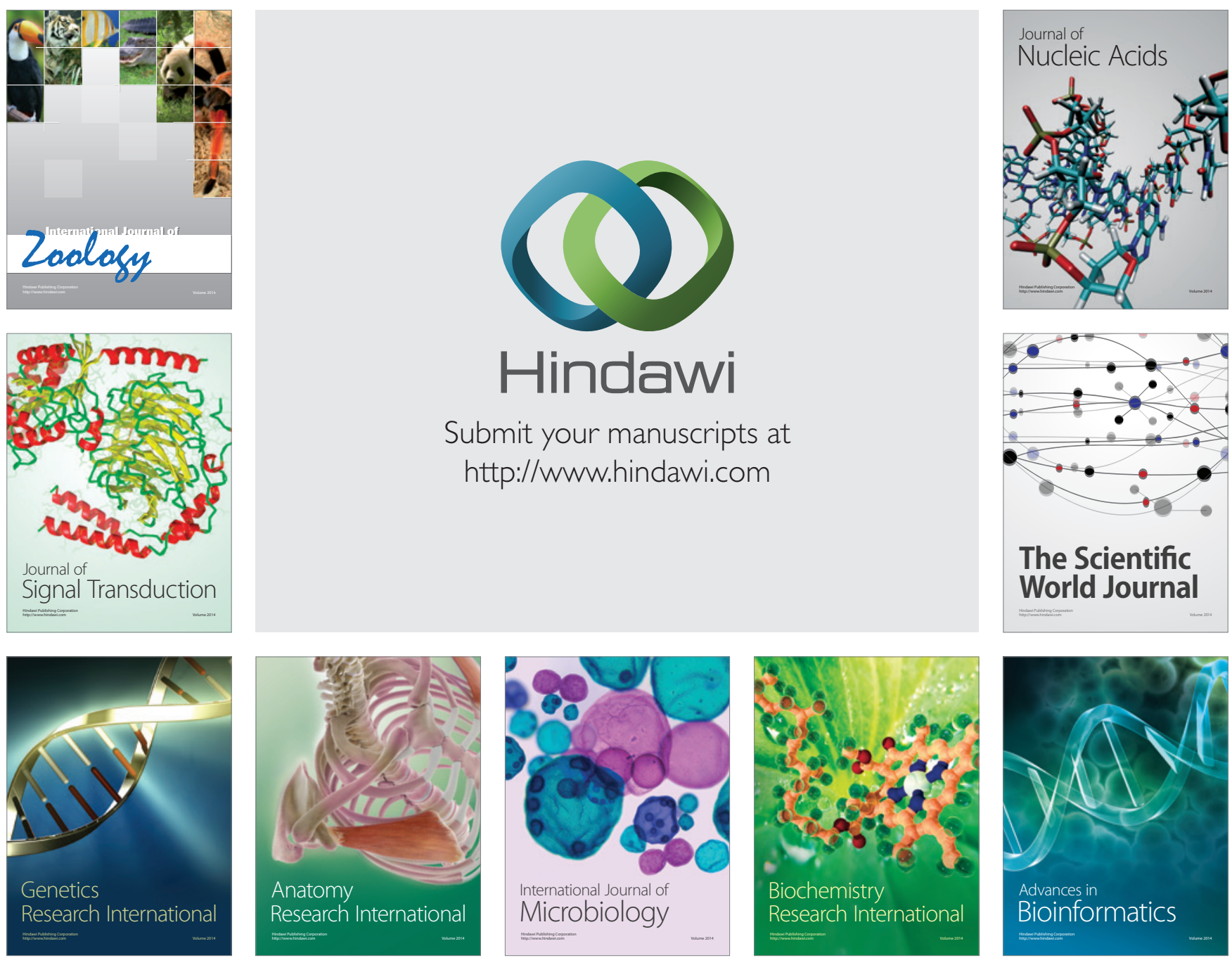

The Scientific World Journal
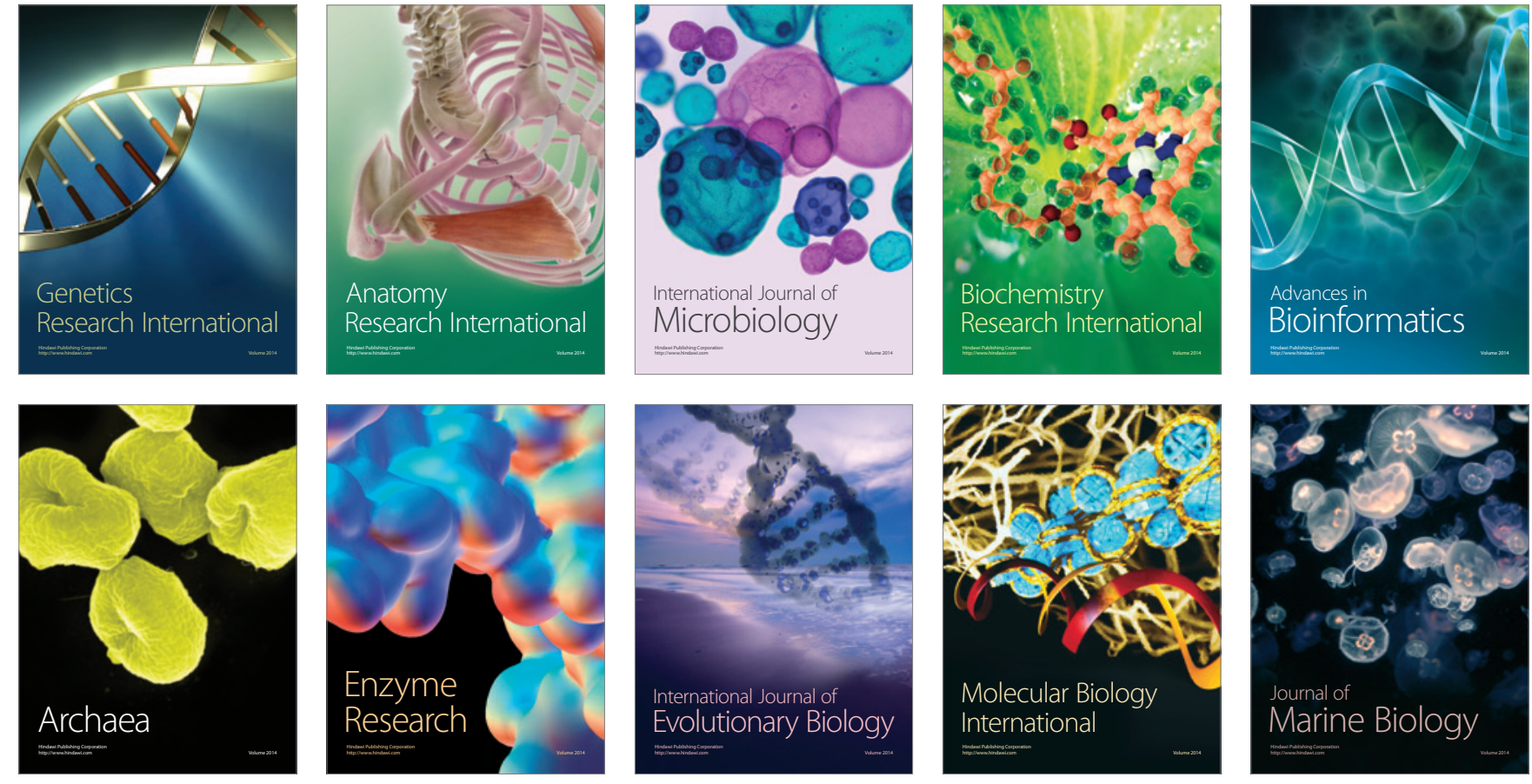